\title{
Critique of "An Electron Microscope Study of Crystal Calcium Carbonate Formation in the Mouse Otolith"
}

\author{
DONALD R. PEACOR, ROLAND C. ROUSE, AND MURIEL D. ROSS \\ Department of Geological Sciences (D.R.P., R.C.R.), and Department of \\ Anatomy (M.D.R.), The University of Michigan, Ann Arbor, Michigan 48109
}

\begin{abstract}
Electron micrographs of otoconia of fetal mice, as obtained by Nakahara and Bevelander, provide morphological evidence that the otoconia consist of both organic material and calcium carbonate (calcite), contrary to their own conclusions. Calcite is an integral component of otoconia, apparently from their inception. The concept of mineralization by calcite of an already developed organic template ("preotolith") is shown to be in error.
\end{abstract}

Nakahara and Bevelander ('79) have recently described their results relating to calcium carbonate formation in otoconia of mice. They present a series of electron micrographs of otoconia, the first of which is from the 15.5day-old fetus and shows what they consider to be the initial growth stage of the otoconia. Subsequent micrographs illustrate otoconial growth stages at 17.5 days, newborn, 1st day, 3rd day, and 7th day after birth. These micrographs document the changes in morphology and size of the otoconia. More importantly, they permit characterization, at least in part, of both the calcium carbonate and the supposed precursor organic material. This in turn provides evidence for the nature of the process by which inorganic material is added to the nascent otoconium.

We should like to point out that some of the crystallographic features of the otoconia have been misidentified by Nakahara and Bevelander. More importantly, certain of the crystallographic relations of the otoconia that they illustrate provide direct evidence for important aspects of the otoconial growth history which are in part at variance with Nakahara and Bevelander's conclusions. As a secondary issue, we also note that their use of certain crystallographic terms is not in accord with accepted usage. As these terms describe significant features of the otoconia, it is essential that they be properly used.
Much of our commentary is concerned with the meaning and significance of the term "single crystal," specifically, single crystals of calcite, $\mathrm{CaCO}_{3}$, which forms the inorganic component of mammalian otoconia. A single crystal of calcite is an object within which the calcium and carbonate ions are ordered in a characteristic array. This array is periodic and continuous in three dimensions. The morphological symmetry of the external faces (or outline) of such a single crystal is a manifestation of that order, and it is essential to realize this. Conversely, the regular external form cannot occur if there is no internal order.

Nakahara and Bevelander note that some authors have demonstrated that mammalian otoconia are single crystals of calcite. They fail to note that this has been elucidated in much more detail by additional studies, namely those of Ross et al. ('76) and Ross and Peacor ('75), the latter paper describing crystal features which give evidence about growth conditions. It is now well established that the normal mammalian otoconium is terminated on each end by three planar faces. The whole set of six faces constitutes a "form" (collection of symmetrically related faces) called the rhombohedron. In a single crystal of calcite, if one face of this rhombohedron form is present, then the other

Received February 4, 1980; accepted March 25, 1980. 
five must also be present. This is a consequence of the periodic and symmetrical crystal structure which is continuous throughout the crystal. Peacor (unpublished) has even shown that the particular rhombohedron involved is $\{1011\}$. (Geometrically there are many possible rhombohedra, which differ in shape but are identical in symmetry.) The symbol $\{10 \overline{1} 1\}$ mathematically defines the specific rhombohedron referred to. These relations are determined by observing the angles at which light is reflected from the faces in an optical goniometer.

Nakahara and Bevelander's interpretation of their electron micrographs is that the otoconia are entirely organic and contain no $\mathrm{CaCO}_{3}$ up to the time of birth. This could not have been determined by the methods used insofar as they were described. Fetal otoconia are notoriously subject to decalcification in fixing and other fluids, as several investigators have noted (Lyon, '55; Ross et al., '76). Moreover, Salamat et al. ('80), using the electron microprobe analytical method and otoconia untouched by extraneous fluids, have demonstrated abundant calcium in fetal rat otoconia. Additionally, electron micrographs of unfixed, unstained fetal otoconia show the rod-like array of electron-dense material Nakahara and Bevelander found in crystals of the 7-day-old mouse. It is our belief that Nakahara and Bevelander actually observed decalcification artifacts in fetal otoconia. An analogous problem occurs in the preparation of bone sections for electron microscopy. Apatite crystallites in bone are readily dissolved by aqueous solutions used in tissue preparation and sectioning (Landis et al., '77; Boothroyd, '64; Thorogood and Gray, '75).

Nakahara and Bevelander refer to the prebirth otoconia as "preotoliths." However, the micrographs of otoconia from the 17.5-day fetus clearly show a regular cyrstallographic outline, indicating that the "preotoliths" are, instead, calcite crystals in the truest sense. Nakahara and Bevelander unfortunately refer to the outline as being "hexagonal" (the latter is a welldefined crystallographic term, inappropriately used in this case). Indeed, the "preotoliths" show the common geometry of nearly mature otoconia with terminal rhombohedron faces, two on each end appearing in the sections. The otoconia which show the "hexagonal" outline actually lie with their three-fold axis of symmetry parallel to the plane of the figure.

The significance of these observations is that the so-called preotoliths in the fetal mouse are not composed only of organic material, as claimed by Nakahara and Bevelander. This fact is evident from their own morphological findings. The "preotolith" morphology is consistent with a continuous calcite structure, which has a substantial organic content. The "preotoliths" are single crystals in every sense of the word.

These observations bear on the basic interrelationship of the organic material and the origin of the inorganic calcite. The coexistence of organic matter and calcite at all growth stages implies a continuous generation of calcite concurrently with the organic material from the earliest fetal stages to the final mature otoconia. It is essential to recognize this relation if the ultimate factors concerning generation of otoconia are to be understood. Certainly, the concept that an organic preotolith serves as a template for deposition of calcite later, after birth, is in error.

Lastly, we note that Nakahara and Bevelander introduce the term "multi-iso-oriented" crystal to describe the structure of mature otoconia. In addition to being something of a strain on the English language, this term is superfluous, since there already exists a standard crystallographic term for the phenomenon the authors are trying to describe. Otoconia are single crystals which contain numerous imperfections in their structures describable simply as a "lineage (or mosaic) structure." This is the common status of crystals in general. They frequently consist of subunits, each of which is relatively perfect internally but which is slightly out of alignment with neighboring subunits. In the case of calcite otoconia, these imperfect subunit boundary regions almost certainly contain high concentrations of organic material.

\section{LITERATURE CITED}

Boothroyd, B. (1964) The problem of demineralization in thin sections of fully calcified bone. J. Cell Biol., 20:165-173.

Landis, W.J., M.C. Paine, and M.J. Glimcher (1977) Electron microscopic observations of bone tissue prepared anhydrously in organic solvents. J. Ultrastruct. Res., 59:1-30.

Lyon, M.F. (1955) The development of the otoliths of the mouse. J. Embryol. Exp. Morphol., 3:213-229.

Nakahara, H., and G. Bevelander (1979) An electron microscope study of calcium carbonate formation in the mouse otolith. Anat. Rec., 193:233-242.

Ross, M.D., and D.R. Peacor (1975) The nature and crystal growth of otoconia in the rat. Ann. Otol. Rhinol. Laryngol., 84:1-15.

Ross, M.D., L.G. Johnson, D. Peacor, and L.F. Allard (1976) Observations on normal and degenerating human otoconia. Ann. Otol. Rhinol, Laryngol., 85:1-17.

Salamat, S., M.D. Ross, and D.R. Peacor (1980) Otoconial formation in the fetal rat. Ann. Otol. Rhinol. Laryngol. (in press).

Thorogood, P.V., and J.C. Gray (1975) Demineralization of bone matrix: Observations from electron microscope and electron-probe analysis. Calcif.' 'Tiss. Res., 19:17-26. 